\title{
Desregulación de la formación inicial docente. Una experiencia neoliberal ${ }^{1}$
}

\author{
Deregulation of initial teacher education. A neoliberal experience \\ Desregulamentação da formação inicial de professores. Uma experiência \\ neoliberal
}

Ilich Silva-Peña ${ }^{2}$
Universidad Católica de Temuco, Chile
César Peña-Sandoval ${ }^{3}$
Pontificia Universidad Católica de Valparaíso, Chile

Recepción: 10/12/2018

Evaluación: 28/12/2018

Aceptación: 17/02/2019

Artículo de Investigación - Revisión

https://doi.org/10.19053/01227238.8724

\begin{abstract}
RESUMEN
El presente artículo plantea el problema de la desregulación de la formación inicial docente bajo un sistema neoliberal. Chile fue utilizado como un laboratorio social de transformación política y económica por un grupo de economistas chilenos formados en Estados Unidos (Chicago Boys). El texto entrega antecedentes históricos del proceso realizado por la dictadura cívico-militar en Chile y continuado por los gobiernos siguientes. Este marco político e histórico permite visualizar el

sistema universitario chileno, en el cual las instituciones estatales son afectadas por un modelo de autofinanciamiento.

En el artículo se enfatiza el análisis en torno a cuestionar la dicotomía regulación/desregulación como una distinción público/privada. Como ejemplo se muestra la expansión de una oferta privada y desregulada de la formación docente por parte de universidades estatales regionales con bajo aporte basal estatal. A través de estos casos es posible observar que un

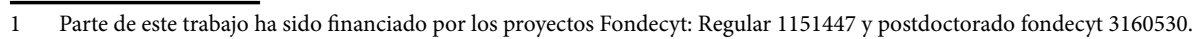

2 Profesor de Educación General Básica y Licenciado en Educación (Universidad Metropolitana de Ciencias de la Educación, UMCE). Doctor en Ciencias de la Educación (Pontificia Universidad Católica de Chile). Durante su formación desarrolló una estancia doctoral en University Wisconsin-Madison. Ha trabajado como académico e investigador en diversas universidades en Chile. Durante 6 años dirigió el hoy desaparecido Instituto de Investigaciones Pedagógicas de la Universidad Arturo Prat. Postdoctorado en el Centre for Research for Teacher Education and Development. Universidad de Alberta. Canadá. Correo electrónico: ilichsp@gmail.com

3 Profesor de Educación Media en Filosofía, Licenciatura en Educación, Licenciatura en Filosofía, Universidad de Chile. Magister en Currículum y Comunidad Educativa, Universidad de Chile. Ph.D. Currículum and Instruction, University of Washington, Seattle, USA. Académico de la Pontificia Universidad Católica de Valparaíso.
\end{abstract}


modelo basado en la oferta y demanda avanza en la destrucción de las fronteras entre lo público y privado. Como buen laboratorio, el ejemplo de Chile puede ser utilizado para observar los efectos de una política pública neoliberal.

Palabras clave: Política educacional; gastos educativos; subvención educativa; Chile; privatización.

\section{ABSTRACT}

This paper addresses the problem of deregulation of initial teacher education under a neoliberal system. Chile was used as a social laboratory of political and economic transformation by a group of Chilean economists trained in the United States (Chicago Boys). The text provides historical background of the process carried out by the civil-military dictatorship in Chile, continued by the following governments. This political and historical framework makes it possible to visualize the Chilean university system, in which state institutions are affected by a self-financing model.

We emphasize the analysis around the regulation/deregulation dichotomy as a public/private distinction. An example is the expansion of a private and deregulated offer of teacher education by regional state universities with low basal state contribution. Through these cases it is possible to observe that a model based on supply and demand advances in the destruction of the frontiers between public and private sectors. As a good laboratory, the example of Chile can be used to observe the effects of a neoliberal public policy. Keywords: Education policy; education expenditures; education subsidy; Chile; privatization.

\section{RESUMO}

Este artigo levanta o problema da desregulamentação da formação inicial de professores sob um sistema neoliberal. $\mathrm{O}$ Chile foi usado como laboratório social para transformação política e econômica por um grupo de economistas chilenos treinados nos Estados Unidos (Chicago Boys). O texto fornece antecedentes históricos do processo realizado pela ditadura cívico-militar no Chile e continuado pelos seguintes governos. Esse arcabouço político e histórico nos permite visualizar o sistema universitário chileno, no qual as instituições do Estado são afetadas por um modelo de autofinanciamento.

$\mathrm{O}$ artigo enfatiza a análise em torno de questionar a dicotomia regulação / desregulamentação como uma distinção público / privado. Um exemplo é a expansão de uma oferta privada e desregulada de formação de professores por universidades estaduais regionais com baixa contribuição de estado de referência. Por meio desses casos, é possível observar que um modelo baseado na oferta e demanda avança na destruição das fronteiras entre o público e o privado. Como um bom laboratório, o exemplo do Chile pode ser usado para observar os efeitos de uma política pública neoliberal.

Palavras-chave: Política educacional; despesas educacionais; bolsa educacional; Chile; privatização. 


\section{INTRODUCCIÓN}

A nivel internacional, la discusión sobre caminos alternativos de formación del profesorado tiene larga data ${ }^{4,5,6}$. En la literatura podemos ver que se identifican diversos puntos de vista en los procesos de regulación/desregulación: los defensores, que defienden la formación docente tradicional y pública; los reformistas, que desean construir un sistema basado en las políticas del mercado; y los transformadores, que reconocen las fortalezas y debilidades de los institutos y universidades públicas que educan a los(as) futuros(as) profesores(as), pensando en la necesidad de construir un nuevo sistema de formación docente de carácter público ${ }^{7}$.

Este trabajo, a través del análisis histórico reciente, sostiene que los procesos de desregulación de la formación docente no deberían centrarse solo en las universidades privadas, sino más bien en el modelo económico por el cual se rigen todas las instituciones de educación superior. La discusión se plantea desde un país en donde la confusión de lo público con lo privado llega a niveles extremos. En Chile, las instituciones estatales (que en rigor debieran ser las más públicas) se rigen por un sistema de subvención que no dista mucho de las instituciones privadas. Este sistema llevó en algunos momentos de la historia a un proceso de desregulación de la formación docente que afectó a todo el sistema. Aunque hoy en día, nuevas regulaciones han revertido esa situación. Lo que se plantea aquí es una propuesta para analizar lo que llevó a este proceso.

La amenaza hacia el sistema de educación superior proviene no solo desde fundaciones o corporaciones privadas que dicen no tener fines de lucro, también desde las mismas universidades públicas. Cuando el autofinanciamiento y la competencia son los modelos prevalentes, la formación pública de docentes se ve enfrentada a los mismos problemas y transformaciones que han regido a las iniciativas privadas. Para ejemplificar este fenómeno, aquí mostramos la forma en que universidades públicas chilenas han utilizado estrategias orientadas al mercado en el área de la pedagogía, convirtiéndose así en líderes en la desregulación del "mercado de la formación docente"

En Chile la Educación superior mutó desde un sistema dicotómico en el que existían universidades estatales y universidades privadas con apoyo estatal a te-

4 Linda Darling-Hamond y Ann Lieberman, Teacher education around the world: Changing policies and practices (New York: Routledge, 2013).

5 Julio Diniz-Pereira, "As licenciaturas e as novas políticas educacionais para a formação docente", Educação \& sociedade, v. 20, no. 68, (1999): 109-125.

6 Ken Zeichner, "The Adequacies and Inadequacies of Three Current Strategies to Recruit, Prepare, and Retain the Best Teachers for All Students", Teachers College record, v. 105(3), (2003): 490-519.

7 Ken Zeichner y César Peña-Sandoval. "Venture philanthropy and teacher education policy in the US: The role of the New Schools Venture Fund", Teachers College record, v. 117, no. 6, (2015):1-24.

8 Para un mayor detalle de las transformaciones en Educación Superior en Chile, ver: Mauricio Rifo Melo (2017). "Historia de la transformación en la educación superior chilena (1973-1990)”. Revista Historia de La Educación Latinoamericana, v. 19, n. 28, (2017), 13-36. doi.org/10.19053/01227238.4925; Alejandra Contreras Gutiérrez. La enseñanza jesuita en Chile colonial: sus colegios, universidades y una aproximación a sus métodos y contenidos. Revista Historia de la Educación Latinoamericana, vol. 16 no. 22 (2014): 35-50. https://doi.org/10.19053/01227238.2687. 
ner hoy múltiples definiciones. La diversidad de modos de financiar las universidades chilenas en la actualidad no permite visualizar claramente hasta donde una institución es pública o hasta donde llega lo privado. Esto ha generado un sistema de Educación Superior diverso con una interacción permanente entre lo privado y lo público?.

Sabemos que la discusión de los límites entre lo que se considera público y privado es compleja, relevante y necesaria. Sin embargo, no es el objetivo de este trabajo entrar en esa discusión. Para efectos de este texto asociaremos lo público a lo estatal que sabemos, es lo que sí o sí debiera ser público. Las instituciones que pertenecen al Estado se financian a través de los aranceles que pagan sus estudiantes y aportes que entrega el Estado, algo que muchas veces no la distingue de instituciones privadas con financiamiento estatal. Esto hace que existan múltiples clasificaciones con un sistema difícil de comprender. En cuanto a las universidades privadas, el sistema actual permite la existencia de aquellas que tienen fines de lucro (aunque la ley lo prohíba, no hay castigo por lucrar), sin fines de lucro financiadas por el Estado, sin fines de lucro no financiadas por el Estado, y dentro de ellas confesionales y no confesionales.

En este texto deseamos hacer visible el efecto de las políticas macroeconómicas al presionar a las instituciones públicas (estatales) hacia el proceso de desregulación. Nuestra pretensión es contribuir a la conversación académica sobre la formación del profesorado en condiciones pro-mercado en las instituciones públicas. Para entregar un ejemplo de las políticas y cambios impulsados por el mercado, nos enfocamos en Chile, donde probablemente se han implementado las políticas de liberalización económica más extremas. Pensamos que este texto puede tener utilidad en países donde las formas de autofinanciamiento están inundando a las universidades públicas.

\section{Chile: laboratorio del neoliberalismo}

Durante la década de los años 50, un grupo de académicos de Estados Unidos buscaban un lugar en el mundo para iniciar un proyecto de una nueva visión de la economía ${ }^{10}$. La idea era seleccionar a un grupo de jóvenes economistas que pudieran reemplazar el paradigma clásico capitalista que se había desarrollado en el mundo occidental. El objetivo era cambiar el modelo económico de un país completo. El nuevo modelo consistía en regulaciones impulsadas por el mercado, a diferencia de un capitalismo protector de la burguesía nacional. Esta nueva visión de la economía implicaba un gran cambio en varios servicios, tanto del gobierno como del mercado. Originalmente, este programa fue propuesto para Paraguay, finalmente se instaló en Chile ${ }^{11}$.

\footnotetext{
9 Idem.

10 Juan Gabriel Valdés, Pinochet's Economists: The Chicago School of Economics in Chile (New York: Cambridge University Press, 1995).

11 Juan Gabriel Valdés, La escuela de Chicago: operación Chile, (Buenos Aires: Grupo Editorial Zeta, 1989).
} 
La primera etapa del proyecto consistía en establecer un acuerdo entre una universidad chilena y la Universidad de Chicago ${ }^{12}$. La oferta inicial se le hizo a la Universidad de Chile, institución que por ser estatal podría ejercer mayor influencia en las transformaciones gubernamentales. Sin embargo, la propuesta no prosperó en esa institución. No obstante, la Pontificia Universidad Católica (PUC) -universidad privada más grande de Chile- quería fortalecer su Escuela de Economía. Valdés ${ }^{13}$, remarca que este intercambio académico se enfocó desde un principio en un punto de vista económico específico. La idea era impulsar una visión liberalizadora, que se extendiera a los diferentes ámbitos de la vida social.

Los destacados investigadores de la Universidad de Chicago, Milton Friedman y Arnold Harberger comenzaron un cuidadoso proceso de reclutamiento de estudiantes abiertos a una posición ideológica específica. Se creó un programa especial de posgrado para alumnos chilenos, dirigido por académicos con profundo conocimiento de la perspectiva liberal de la economía ${ }^{14}$. Este grupo, apodado como los "Chicago Boys" tuvo su mayor influencia en la construcción de la estructura económica de la dictadura cívico-militar dirigida por Augusto Pinochet (1973-1990).

El documento clave elaborado por los "Chicago Boys" fue "El ladrillo"15. Dicho trabajo se completó a comienzos de los años 70 y contenía las ideas económicas más importantes para la transformación de la sociedad chilena. Ese documento requería y proponía medidas para instalar al mercado como el regulador de las distintas áreas de la sociedad, afectando también a la educación, previsión social y la salud. Los cambios radicales se produjeron en Chile una vez que el gobierno dictatorial estuvo instalado, afectando toda la economía del país y sus instituciones. La dictadura cívico-militar fue terreno fértil para la instalación de un gran laboratorio social y económico ${ }^{16}$.

El gobierno de los Estados Unidos otorgó permanente apoyo a este proceso. Se otorgaron fondos especiales para la creación de este grupo llamado "Chicago Boys". Los documentos desclasificados de la Agencia Central de Inteligencia (CIA) agregan mayor información respecto a la intervención de Estados Unidos en Chile: El golpe militar para acabar con el régimen de Allende e instalar al General Pinochet como jefe de Estado fue concertado y ejecutado desde la potencia del norte ${ }^{17}$.

En 1980 fue el momento en que a través de una alianza entre la derecha política que apoyaba la dictadura y este grupo de economistas comenzaron una serie de transformaciones políticas, económicas y sociales. Los civiles que fueron par-

12 José Ossandón. "La escuela de chicago - una mirada histórica a 50 años del convenio Chicago/ Universidad Católica: ensayos en honor a Arnold c. Harbeger". Universum (Talca), v. 25, no 2 (2010): 217-220.

13 Op. cit

14 María Olivia Monckeberg, Privatización de las Universidades (Santiago: Copa Rota, 2005).

15 Sergio De Castro. El Ladrillo: bases de la política económica del gobierno militar chileno (Santiago: Centro de Estudios Públicos, 1992).

16Manuel Délano y Hugo Traslaviña, La herencia de los Chicago Boys (Santiago: Ornitorrinco, 1989).

17 Peter Kornbluh, “The Declassified Pinochet File: Delivering the Verdict of History”, Radical history review, no. 124 (2016): 203-216. 
te de la dictadura iniciaron un análisis sobre el modelo económico que debía ser instalado. Su objetivo era convencer al poder militar, especialmente a Pinochet, para realizar los cambios que ellos consideraban necesarios. Al comienzo de la dictadura no existía una comprensión desde la derecha conservadora sobre las ideas económicas de los Chicago Boys ${ }^{18}$. Los equipos políticos logran convencer al poder militar y se establece un nuevo marco legal, en el año 1980 se instala una nueva constitución. Sobre la base de este nuevo marco legal se generaron grandes cambios económicos. En el área de la educación la nueva transformación tuvo un enorme impacto ya que debilitó el sistema público promoviendo proyectos empresariales en los distintos niveles de la educación chilena ${ }^{19}$.

Al cambio generado en los sectores sociales se agregó una acelerada privatización de los activos públicos. Uno de los más claros ejemplos de enajenación fue la privatización de las compañías estatales. Se privatizaron los servicios esenciales de agua, electricidad, telecomunicaciones y correos ${ }^{20}$. Aunque este proceso comenzó bajo la dictadura, no se detuvo con el término del régimen de Pinochet. Por el contrario, las iniciativas de privatización continuaron durante los gobiernos de la coalición de partidos democráticos (la Concertación), especialmente durante los años $90^{21}$. A principios de siglo nos encontramos con una privatización del país casi total.

\section{Las transformaciones en educación}

Las transformaciones neoliberales en el sistema educativo incluyeron un proceso de municipalización bajo la excusa de la descentralización. Esto consistía en traspasar la tuición de los establecimientos educacionales de Educación Básica y Media desde el Ministerio de Educación a los municipios. La transferencia mantenía en manos públicas la educación escolar, sin embargo, se agregó la figura de las "Corporaciones Municipales", organismos privados que administran de modo independiente los establecimientos. Esto era el paso previo a la privatización total del sistema de educación ${ }^{22}$.

Durante ese mismo periodo se comienza a implementar el sistema de "voucher" propuesto por Milton Friedman. Este mecanismo proponía que los(as) estudiantes actuaran como portadores del financiamiento. El sistema se desarrolló tanto para los establecimientos municipales como para los privados ${ }^{23}$. Esto significó crear una figura nueva, los colegios particulares subvencionados, colegios en manos privadas que cuentan con financiamiento estatal. Este sistema consi-

\footnotetext{
18 Monckeberg, María Olivia. Con fines de lucro. Santiago: Penguin Random House, 2013.

19 Op. Cit.

20 Para profundizar más acerca de este proceso puede ver: Monckeberg, María Olivia. "El Saqueo de los Grupos Económicos al Estado Chileno y Verdad Oculta de las Empresas Públicas." Santiago, Chile: Editorial Ediciones B (2001).

21 Jorge Nef, "The Chilean model: fact and fiction", Latin American perspectives, v. 30, no. 5, (2003) 16-40.

22 Op. cit.

23 Solo quedaron un 7\% del total de la matrícula correspondiente a colegios completamente privados. Los colegios privados que educan a la élite económica y política del país se mantienen fuera del financiamiento vía subvención.
} 
dera la matrícula y la asistencia diaria para el cálculo de los aportes. El financiamiento vía voucher se implementó tanto para las escuelas privadas subvencionadas como para las municipales. Esta vía para incorporar los aportes representa la esencia del "Estado Subsidiario", un concepto inscrito en la constitución de 1980 que reemplazó al de "Estado Docente" que había sido parte de la historia de Chile desde hace más de un siglo. Esta visión económica convirtió a la educación en un bien transable en el mercado ${ }^{24,2526}$.

En el caso de la educación superior, durante los años 80 , se procedió a un desmembramiento de las principales universidades estatales. A partir de las sedes de las Universidades de Chile y Técnica del Estado fueron creadas universidades regionales. Las universidades estatales contaban con un presupuesto anual específico que comenzó a depender de la cantidad de matrículas y aranceles de sus estudiantes. Esta transformación cambia el orden anterior a los años 80 en el que las universidades privadas eran sin fines de lucro. El nuevo orden mantuvo a las instituciones formadas antes de los $80 \mathrm{y}$ sus derivadas, las que siguen operando hasta hoy como parte del Consejo de Rectores de Universidades Chilenas (CRUCH), incluyendo a universidades estatales y privadas. A partir del nuevo concepto de educación se crean nuevas universidades privadas, las cuales ampliaron la "oferta" de la educación superior. En menos de una década la transformación del mapa de Educación Superior en Chile fue profunda. Las instituciones estatales pasaron a depender de un sistema de financiamiento que cada vez se asemejaba más al de las universidades privadas. Las universidades públicas perdieron respaldo financiero y se vieron forzadas a sobrevivir en un sistema de autofinanciamiento.

Los sectores socioeconómicos que anteriormente no tenían acceso a la educación superior fueron el público objetivo de la mayoría de las nuevas instituciones privadas. Décadas más tarde, la oferta en la educación superior ha permitido a muchas primeras generaciones de alumnos obtener un título de educación superior, pero en un contexto de desregulación que genera una sobreoferta de profesionales y técnicos en diferentes áreas.

A principios del siglo XXI comenzaban las críticas a los gobiernos concertacionistas señalando que "no incidieron en forma significativa sobre el modelo neoliberal de carácter hegemónico, sumando la lógica del neoliberalismo en sus proyectos coyunturales de modernización ${ }^{\prime 27}$. Estas críticas serían ratificadas con mayor apoyo empírico posteriormente ${ }^{28}$. Hace unos años, utilizando datos de la OCDE ${ }^{29}$ se evacuaron reportes considerando a Chile como uno de los países

24 Jorge Nef. "El concepto de Estado subsidiario y la educación como bien de mercado: Un bosquejo de análisis político" Enfoques educacionales, v. 2, (2007).

25 María Angélica Oliva. "Política educativa y profundización de la desigualdad en Chile”. Estudios Pedagógicos (2008) 207-226.

26 José Bravo (2013). Educación pública, autonomía universitaria y cambio político: notas para el análisis del movimiento universitario en chile, 2011. Revista Historia de la Educación Latinoamericana, 15 (21), 263-282.

27 Maximiliano Soto, "Políticas Educacionales en Chile durante el Siglo XX", Revista Mad, v. 0, no. 10 (2004) p. 4.

28 Cristian Bellei, El gran experimento. Mercado y privatización de la educación chilena, (Santiago: LOM ediciones) 2015.

29 OCDE, "Establecimiento de un marco para la evaluación e incentivos docentes. Consideraciones para México" (París: OECD) 2011. 
que tiene el mayor porcentaje de escuelas privadas en el mundo ${ }^{30}$. Mientras que en la OCDE existe un promedio de $80 \%$ de la educación en manos del sector público, en el caso de Chile dicha cifra aún no supera el $44 \%{ }^{31}$. Además, en los últimos años se ha generado el cierre de escuelas públicas que agravan la situación de las comunidades en dichos establecimientos ${ }^{32}$. Tanto la privatización del sector escolar como el proceso en la Educación Superior son muestras del éxito del experimento de instalación de un modelo neoliberal en Chile ${ }^{33}$. El proceso de transformación tuvo un carácter despiadado durante la Dictadura ${ }^{34}$ y fue fortalecido en los años de gobierno de la concertación. Las críticas a este modelo de financiamiento fueron la base fundamental para las críticas del movimiento social por la educación en los últimos años ${ }^{35,36,37}$.

\section{El proceso de desregulación y la lucha de las universidades por el financia- miento}

En la década de los 90, cuando estaba por finalizar el periodo del dictador Augusto Pinochet, fue el momento de creación de la mayor cantidad de nuevas universidades privadas. El último día de la dictadura cívico militar, el 10 de marzo de 1990, se promulgó la Ley Orgánica Constitucional de Enseñanza (LOCE) que regulaba todos los niveles de educación. Con esto se sellaba un marco institucional para la transformación neoliberal realizada. En el caso de la Educación Superior, la creación de universidades privadas estuvo liderada por quienes fueron miembros vinculados con la dictadura ${ }^{38}$. En años posteriores, se incorporaron a los directorios de este tipo de universidades, miembros de sectores proclives al nuevo gobierno. De esta forma, comenzaron nuevos vínculos en el seno de los directorios de dichas universidades.

Por ley, las universidades chilenas son organizaciones sin fines de lucro; sin embargo, los grupos económicos que se ubican tras estas instituciones privadas poseen diversas formas de generar ganancias. Una de las fórmulas; las empresas espejo, figura que consiste en tener una empresa que es propiedad de las mismas personas involucradas en las corporaciones sin fines de lucro (universidades). Estas entidades proveen servicios tales como limpieza y seguridad o son

30 Marco Kremerman. El desalojo de la educación pública. Serie “Ideas para el buen vivir”, Fundación SOL. (2011).

31 Mineduc. Estadísticas de la educación 2016. Santiago: Mineduc, 2017.

32 Mauricio Pino-Yancovic. "Parents' defense of their children's right to education: Resistance experiences against public school closings in Chile", Education, Citizenship and Social Justice, v. 10, no. 3, (2015) 254-265.

33 María Olivia Monckeberg. Privatización de las Universidades (Santiago: Copa Rota) 2005.

34 Ana López. "De la dictadura militar a la democracia pactada de la Concertación y la derecha: El modelo de la Universidad-Empresa en Chile (1973-2008)", Lucha de clases, v. 1, 2011.

35 Cristian, Bellei; Cristian, Cabalin. "Chilean Student Movements: Sustained Struggle to Transform a Market-Oriented Educational System”. Current Issues in Comparative Education, v. 15, no. 2, (2013) 108-123.

36 Cabalin, Cristian. "Neoliberal Education and Student Movements in Chile: Inequalities and Malaise". Policy Futures in Education, v. 10, no. 2, (2012) 219-228.

37 Vicente Sisto. "Entre la Privatización y la Reconstrucción de lo Público en Chile: Movimientos Estudiantiles y el Debate acerca del Devenir de la Universidad”. Horizontes Sociológicos, v. 0, no. 1, (2015) 57-63.

38 María Olivia Monckeberg. Con fines de lucro (Santiago: Penguin Random House) 2013. 
del ámbito inmobiliario que arrienda edificios a la universidad. A este sistema de financiamiento se suma la exención de impuestos a las que se someten este tipo de instituciones. Así, logran mantener sus negocios gracias a un sistema de educación superior desregulado. En un comienzo, se permitió la creación de universidades contando con mínimos recursos, generando carreras que no tenían ninguna proyección laboral debido a la sobreoferta. Además, algunas universidades se instalaron en viejos edificios que fueron arrendados para esos propósitos; sus bibliotecas se desarrollaron sobre la base del uso de fotocopias, faltando a las normas de propiedad intelectual, es decir, desregulación total ${ }^{39}$.

Las universidades públicas también debían adaptarse a la lógica del autofinanciamiento. Por lo tanto, se organizaron para aprovechar el contexto desregulado y sobrevivir a este. Una ley creada durante la dictadura estableció la limitada ayuda estatal para las universidades del CRUCH (creadas antes de 1980), instituciones tanto privadas como públicas sin fines de lucro. Varias de ellas eran universidades regionales derivadas de las dos universidades desplegadas a nivel nacional (U. de Chile y PUC). Las más nuevas recibían un menor financiamiento estatal que las más antiguas. Entre las universidades regionales que recibían un menor porcentaje de financiamiento estaban la Universidad de Los Lagos (ULA) cuya sede central se ubicaba en Osorno (sur de Chile) y la Universidad Arturo Prat (UNAP), con su rectoría ubicada en la ciudad de Iquique (norte de Chile). Dichas universidades fueron inicialmente sedes de la Universidad de Chile, en los años 80 pasaron a ser institutos profesionales, para luego convertirse en universidades regionales. Estas son las últimas universidades estatales creadas durante la dictadura. A todas estas instituciones se les aseguró un mínimo de financiamiento basal. Aunque dicho financiamiento, en algunos casos, no alcanzaba a cubrir el $15 \%$ del total de gastos de la misma.

La necesidad de autofinanciamiento y la desregulación instalada en el sistema de educación superior de Chile amplía la oferta de carreras universitarias permitiendo una mayor inyección de recursos financieros a las universidades. Consecuentemente, varias universidades crearon proyectos alternativos a la formación de profesores y profesoras. Las universidades que generaron una mayor cantidad de matrículas fueron dos estatales (ULA y UNAP), es decir, las últimas en ser creadas por la dictadura y que contaban con el menor financiamiento del Estado. Ambas comenzaron a ofrecer certificaciones alternativas para pedagogía. Esta nueva "oferta académica" fue la respuesta al sistema de autofinanciamiento que regía tanto para las instituciones públicas como privadas. Destacamos estos dos casos ya que ambas son universidades estatales que tienen un rol relevante en los procesos de desregulación de la formación docente. 


\section{La fábrica de profesores}

En su libro "La privatización de las Universidades: una historia de dinero, poder e influencia", Monckeberg ${ }^{40}$ acuñó el término "fábrica de profesores". De esta forma, se refería al proceso de formar una cantidad excepcional de docentes en pocos años. De hecho, durante la década del 2000, las universidades que ofrecían programas de pedagogía tuvieron un crecimiento desmesurado. A mediados de los años 90, una reforma educativa aumentó las horas de la jornada escolar en las escuelas chilenas, lo que produjo una mayor demanda de docentes. Además, debido a que en este período un gran número de profesores en ejercicio no tenían el título universitario, se crearon programas alternativos de formación para regular los títulos de pedagogía. Hasta entonces, parte del personal docente no tenía una formación universitaria en educación ni contaba con un permiso especial del Ministerio de Educación para ejercer. La mayoría de quienes pertenecían a este grupo de docentes sin certificación trabajaban a tiempo completo en escuelas rurales o de bajos ingresos.

El actual sistema universitario, basado en las leyes dominantes que impone el neoliberalismo económico, promovió una reacción de las universidades tradicionales de acuerdo con las mismas lógicas del mercado. Instituciones educativas, que durante mucho tiempo se habían destacado por su visión altruista, comenzaron a mutar para poder subsistir. El camino de las instituciones estatales ha sido el desarrollo de una serie de fuentes alternativas de financiamiento. Dentro de estas acciones se han implementado "programas especiales de titulación, capacitación y asesorías a empresas; llegando incluso, algunas casas de estudio, a arrendar sus dependencias". Las instituciones de educación superior que ofrecen pedagogía han reconocido que los programas especiales de titulación fueron parte de las medidas para el sistema de autofinanciamiento ${ }^{41}$. Estos programas eran fáciles de implementar y la necesidad de recursos humanos en las escuelas ejerció presión para la formación masiva de docentes. Los programas alternativos se realizaban durante las vacaciones de invierno y verano para profesores en ejercicio sin certificados que debían cumplir con las nuevas condiciones legales de tener un título universitario para ejercer. Quienes fueron formados en estos programas se prepararon en una época donde no existía un sistema de acreditación de carreras, por lo que la calidad de esa preparación es, al menos, dudosa, considerando la diferencia en cantidad de horas y modalidad de formación ${ }^{42,43}$.

40 Ibídem.

41 Francisco Ganga y José Vera. "Reflexiones en torno al nuevo escenario de la gestión universitaria”, Cuadernos de la Facultad de Humanidades y Ciencias Sociales. Universidad Nacional de Jujuy, n. 30, (2006) 41-61.

42 Andrea Ruffinelli. Actualización de la sistematización de la oferta de Programas Especiales de Pedagogía en Educación Básica de las Instituciones de Educación Superior Chilenas Resumen Ejecutivo. (Santiago: CIDE, Universidad Alberto Hurtado) 2007.

43 Andrea Ruffinelli y Luis Sepúlveda. Sistematización de la oferta de programas especiales de pedagogía en educación básica de las instituciones de educación superior chilenas. (Santiago: CIDE, Universidad Alberto Hurtado) 2005. 
La información acerca de los programas mencionados nunca ha sido pública. Por ejemplo, no existe claridad en relación al número de estudiantes y el monto del financiamiento que las universidades recibieron por esta vía. Sin embargo, hay claridad acerca de la operación de dichos programas, por ejemplo, en términos de su pobre infraestructura. Generalmente, había una sola oficina en un pueblo pequeño y salas de clases de alguna escuela municipal que las universidades arrendaban para la formación durante algunos días en la noche o los fines de semana. El sistema de costos de dichos programas también se basaba en la contratación casual. La incorporación de docentes era exclusivamente en función de determinados cursos de pregrado. Este fue un modelo replicado de las universidades privadas en Chile que trabajan bajo la lógica de contratación temporal (profesores taxis) ${ }^{44}$.

Hoy es muy difícil encontrar información oficial sobre los números de "campus" que estas instituciones llegaron a tener. Sin embargo, existe información de prensa sobre 42 ciudades que contaban con oficinas de universidades que ofrecieron este tipo de formación ${ }^{45}$. La sospecha sobre la mala calidad de estos programas tuvo un impacto en la creación del proceso de acreditación universitaria obligatoria para las carreras de pedagogía. Informes de acreditación ${ }^{46}$ establecen explícitamente que solo algunos campus tenían el potencial para mantener sus programas de formación. Esto indica que el resto no cumplía con los requisitos mínimos de calidad, lo cual pone un manto de duda razonable sobre la calidad de los procesos de formación de quienes egresaron de dichas sedes. Aún así, se debe tener cuidado en estigmatizar a un grupo de docentes provenientes de entornos difíciles y que accedió a la formación que tuvo al alcance en ese momento. Aunque los antecedentes pueden indicar que algunos docentes egresaron de programas alternativos, en estricto rigor, no existen antecedentes que den cuenta de un diferencial cualitativo entre los procesos de formación tradicional y los que se generaron en los programas especiales aludidos. Además, la amplia diversidad de campus y condiciones en las distintas universidades, e incluso al interior de las instituciones, hace que un juicio generalizador quede sin fundamento $^{47}$.

Los programas especiales respondieron a las necesidades del mercado (demanda de más docentes). Bajo un contexto de desregulación, estos docentes sin

44 Daniel Reyes León y Marcelo Santos. "LOS PROFESORES TAXIS-El hedor de lo heredado: La desprofesionalización académica y su parque humano” Le Monde Diplomatique. Edición Chilena 2011.

45 http://www.mercuriocalama.cl/prontus3_mundo_estudiantil/site/artic/20040818/pags/20040818191023.html

46 Informe de Acreditación emitido en el 2006.

47 Es importante destacar que un aspecto es el de la calidad de profesional egresado(a) y otra de la calidad medida por los sistemas de acreditación. El supuesto que existe es que un sistema de calidad nos entregará una formación de calidad y por ende buenos/ as profesionales. En ese sentido, el sistema de acreditación de las universidades en Chile ha ido evolucionando. Hoy en día existen universidades completas que han sido cerradas por no cumplir con ciertos estándares. Más allá de poner en discusión los criterios utilizados para los procesos de acreditación, el sistema ha permitido ir regulando la formación profesional. En el caso de la formación docente, hoy en día tiene una serie de regulaciones que incluso algunos/as académicos/as han considerado como de excesivo control. 
licencia (no certificados) requerían pronto sus títulos profesionales. Las universidades que promovieron la creación de programas de pedagogía a distancia, o solo el día sábado, fueron las opciones para este "mercado". Las instituciones involucradas en esta "oferta" fueron tanto de pertenencia estatal como privada. Los antecedentes recogidos por Ruffinelli y Sepúlveda (2005) señalan que fueron 20 las universidades que generaron este tipo de programas. En la siguiente sección nos centramos en dos casos, ambas universidades estatales que tuvieron mayor cobertura en estos programas.

Los programas que no fueron acreditados entraron a un proceso de conversión de edificios universitarios a Institutos Profesionales (nivel no universitario), lo cual permitió superar sus problemas económicos. Así las universidades pudieron continuar su desarrollo institucional.

\section{Las universidades estatales}

Dado que las instituciones de educación superior se concentran en tres regiones del país ${ }^{48}$, una gran cantidad de estudiantes de otras regiones deben trasladarse a aquellas para obtener un título profesional. En el caso de la formación de docentes, las sedes universitarias regionales ayudaron a contrarrestar la centralización de la oferta educativa y ofrecieron oportunidades de educación a personas trabajadoras en lugares más remotos del país. Sin este tipo de programas, muchas personas no hubiesen tenido la posibilidad de acceder a un título universitario. Las instituciones que se constituyeron como vanguardia en la generación de estos programas de formación fueron dos pequeñas universidades estatales que recibían el menor porcentaje del presupuesto destinado a la Educación Superior. Estas eran la Universidad de Los Lagos (ULA) y la Universidad Arturo Prat (UNAP).

La Universidad de los Lagos (ULA) surge a partir de la fusión de una sede de la Universidad de Chile (Osorno, sur de Chile) y otra de la Universidad Técnica del Estado (Puerto Montt, también al sur). Su campus principal se ubica en Osorno, a 830 kilómetros de Santiago. Al igual que otras universidades estatales, la ULA se ha vio afectada por los problemas que se derivan del auto-financiamiento. En el caso de la Universidad Arturo Prat (UNAP), se trata de una universidad estatal regional ubicada en Iquique, a unos 1750 kilómetros al norte de Santiago. Sus orígenes se encuentran en una sede regional de la Universidad de Chile una vez que esta fue desmantelada a principio de los 80 . Al igual que la ULA, la UNAP fue una de las universidades más afectadas en la distribución de recursos.

48 Según el informe CNED (Consejo Nacional de Educación) El 70\% de la matrícula de Educación Superior está concentrada en las regiones Metropolitana, Valparaíso y Bío Bío. En el caso de la Región Metropolitana concentra el 46, 2\%. Ver: http://www.cned.cl/ indices/matricula-sistema-de-educacion-superior 
Tras un proceso de crisis interna en los años 90, la UNAP logró expandirse con una sede en la ciudad de Victoria, región de la Araucanía, a 2.300 kilómetros del campus principal en Iquique. Producto de una exigencia interna de autofinanciamiento, este campus tuvo dificultades económicas que se mantuvieron por largo tiempo. Como una manera de sobrellevarlos, la UNAP estimuló un programa de pedagogía para certificar a los docentes en ejercicio que no tenían título profesional en una región con altas necesidades educativas como la Araucanía ${ }^{49}$.

Los proyectos de la UNAP y la ULA fueron ejemplos emblemáticos del proceso de desregulación, en especial por el volumen de matrícula que manejaron. Desafortunadamente, es difícil estimar con exactitud la cantidad de estudiantes que participaron en aquellos programas de pedagogía. Aun así, algunos datos disponibles indican que la ULA aumentó su matrícula entre 2004 y 2005 en un $383 \%$. En el caso de la UNAP, los datos representan un incremento del $263 \%$ en el mismo periodo ${ }^{50}$. Al mismo tiempo, se expresa la dificultad para acceder a datos exactos sobre esta explosión de estudiantes de pedagogía, tanto en estas dos universidades como en las otras 18 que tuvieron programas similares ${ }^{51}$.

Para el Estado, estos programas alternativos ofrecieron solución a dos problemas simultáneamente. Por una parte, resolvieron el problema del financiamiento de instituciones menoscabadas a la hora de construir los procesos de distribución del financiamiento basal. Quienes ingresaban a estos programas especiales debían pagar sus colegiaturas en efectivo o con préstamo universitario sin acceso a las garantías que ofrecían los programas de carácter regular con acceso a través de las pruebas estandarizadas. Esto permitió recaudar recursos frescos para la institución. Por otra parte, estos programas ayudaron a suplir la necesidad de dotación profesional para el sistema educacional. A mediados de los años 90 comenzó una reforma educativa que aumentaba la jornada escolar (conocida como JEC), esto significó la necesidad de contar con una mayor planta en las instituciones educativas.

Aunque esta forma de resolver el problema de la escasez docente fue criticada en algunos informes oficiales ${ }^{52}$. La posibilidad de autofinanciamiento que generaron estas vías alternativas permitió que el Estado se desentendiera, durante un buen tiempo, de dos de las universidades con menores recursos. Una interpretación posible a esta actitud pasiva de quienes lideraban el Ministerio de Educación en la época podría ser la posibilidad de controlar posibles focos de protestas generados en dichas instituciones. De este modo se mantiene un

49 Andrés Bravo. "Historia y gestión del asentamiento de la Universidad Arturo Prat en la ciudad de Victoria" (Documento de trabajo), 2011.

50 Op. Cit.

51 Tal como lo hemos expresado, fueron muchas las universidades que implementaron programas de este tipo. Tanto universidades privadas con y sin financiamiento estatal, como solo estatales.

52 Para profundizar más se puede recurrir al Informe comisión sobre formación inicial docente. (CPEIP) 2005. 
control a través de la permisividad y, a la vez, se avanza en la privatización de universidades estatales.

Las universidades estatales involucradas en este tipo de formación docente agudizaron una situación que ya era problemática. La mayor crítica a este fenómeno radica en la falta de rigor en la creación de estos programas, la escasez de infraestructura, bibliotecas con material de baja calidad (un punto clave para las sedes recientemente creadas). Esto se contradice con la creencia tradicional de que la universidad motiva la investigación, la educación y la vinculación con el medio; por el contrario, el foco de estas instituciones solo se mantuvo en entrenar profesores de la manera más rápida y rentable posible. La necesidad de contar con académicos(as) para la formación nuevos(as) profesores(as) requirió la rápida contratación de personal que no contaba con experiencia en docencia o investigación educativa. La mayoría de los docentes contratados eran profesores de establecimientos escolares que, si bien se buscaban docentes con alto desempeño en los espacios escolares, no hay garantías de un impacto en una docencia de calidad en el nivel universitario.

Estos procesos de privatización encubierta y desregulada de las escuelas de pedagogía fueron parte del aumento de la matrícula en el área. En el estudio de actualización acerca de programas de pedagogía realizado por Ruffinelli (op. cit.), de las 20 instituciones de educación superior que ofrecían este tipo de pedagogías, al menos la mitad pertenecía a universidades financiadas por el Estado. El aumento de estos programas se estimó entre 225 en 1999 a 698 hasta el $2008{ }^{53}$. Como consecuencia, la tasa de egresados aumentó considerablemente.

\section{CONCLUSIÓN}

En los procesos de formación docente se plantea, muchas veces, la dicotomía regulación/desregulación paralela a lo público/privado. Mirando la historia reciente de Chile, podemos ver que estas distinciones tienen una mayor complejidad. Los procesos de desregulación o regulación están dados por el sistema mayor de financiamiento que rige a las instituciones de educación superior.

Como ya ha sido documentado en una gran cantidad de artículos y libros, Chile fue considerado como un laboratorio de políticas neoliberales por largo tiempo. Como buen laboratorio, ha servido de ejemplo para construir políticas en otros países. El modelo neoliberal ha implementado sistemáticamente políticas de desregulación o, en otras palabras, una regulación a través del mercado (oferta y demanda). Este contexto facilitó el "negocio de la educación" en las universidades. Se permitió a través de diversos mecanismos aumentar las ganancias de quienes eran propietarios de las universidades privadas (empresa-

53 Op. Cit. 
rios ligados a la dictadura cívico-militar y posteriormente incluyeron a políticos ligados a la Concertación de Partidos por la Democracia).

Hemos tratado de revisar históricamente que, en el caso de Chile, el contexto de desregulación no solo impactó a las universidades privadas, sino que también a las instituciones Estatales. Como un modo de resolver problemas de financiamiento, universidades, tanto privadas como tradicionales, crearon nuevas sedes regionales. Algunas de las nuevas sedes mostraron un crecimiento explosivo en la matrícula, entregando títulos universitarios a muy bajo costo a través de programas especiales. La implementación de estas carreras requería una baja inversión. Los principales programas que se dictaron bajo esta modalidad fueron las carreras de Educación básica y parvularia.

Hay poca información que nos permita revelar las causas del crecimiento inorgánico de la matrícula en pedagogía. Sin embargo, podemos plantear algunas hipótesis. Durante mucho tiempo (al menos desde el año 1991 hasta el 2005) no existieron críticas a los modelos de formación docente. Tal vez, porque servía para suplir el financiamiento que dejó de entregarse por la vía regular. Desde el Estado se hacía necesario mejorar el financiamiento de instituciones estatales más desvalidas y a la vez aumentar la cantidad de docentes necesarios para cubrir las horas lectivas en los colegios a partir de la reforma educativa de la jornada escolar completa (JEC). Por esta razón es que planteamos considerar como una lección la experiencia de desregulación chilena, puesto que esta se reafirma en las instituciones del mismo Estado. La implementación de las carreras de pedagogía consideró un mínimo de infraestructura, la cual era arrendada (en muchas ocasiones solo por un día a la semana). Los costos de personal docente fueron reducidos a través de una contratación parcial que permitía agilizar los procesos de vinculación y desvinculación. Miles de profesores y profesoras se formaron bajo este modelo.

Hoy en día se han implementado diversas propuestas para constituir un marco regulatorio de la calidad educativa, en especial de las carreras de educación y salud. Aun así, la preocupación fundamental de este artículo ha sido cómo el modelo económico alcanza no solo a las universidades privadas, sino que también a instituciones que son parte del Estado. En el caso de Chile, la mayor expansión fue justamente en instituciones estatales, aquellas que recibían los menores aportes basales del financiamiento gubernamental. Esta mirada no contradice la dicotomía regulación/desregulación como una distinción público/ privada en términos de instituciones, sino que propone una discusión en términos de cómo las formas de financiamiento de la educación superior inciden en estos procesos de regulación.

En el caso de Chile, el país completo opera bajo la lógica del libre mercado, un neoliberalismo extremo. Aunque existen programas de apoyo a estudiantes o instituciones tratando de mitigar la visión de la educación como un negocio, 
la fórmula de sostenimiento económico sigue siendo el autofinanciamiento. Esta forma de obtener financiamiento es similar tanto para las universidades del mundo privado como para las estatales. La educación desregulada tomó lugar cuando el Estado así lo incentivó a partir de los 80. En el caso de la formación docente, los programas especiales aliviaron la necesidad de cobertura, pero persisten las dudas respecto a su calidad. Se privilegiaron los bajos costos sin un foco en los procesos formativos pedagógicos necesarios.

Este artículo se aparta tanto de la defensa a la formación tradicional de formación docente como de quienes plantean una reforma de flexibilización neoliberal. Más bien, abogamos por una transformación del sistema universitario, que se adapte a las necesidades de formación que tengan los sistemas educativos, siempre que se procure generar programas de pedagogía fuertes con un financiamiento basal importante. Planteamos una política de largo plazo para la formación del profesorado, donde la calidad (no el mercado) de la formación pedagógica sea la prioridad. La construcción de una fuerte formación docente universitaria es prerrequisito para una escuela pública fuerte. Los procesos de regulación/desregulación no son equivalentes a la dicotomía público/privada, dependen de la visión de financiamiento que se establezca para todo el sistema de educación superior. Tal como lo planteamos en un comienzo, Chile fue elegido como un país para construir un modelo económico nuevo. Como todo laboratorio nos sirve para aprender lo que debemos o no hacer en una escala mayor. Lo que ha sucedido en Chile permite tomar lecciones para construir el rumbo de otras naciones, en especial para aquellos países que hoy están en un proceso de debilitamiento de la educación pública en función de la privatización del sistema a través de las políticas de autofinanciamiento.

\section{REFERENCIAS}

Bellei, Cristian. El gran experimento. Mercado y privatización de la educación chilena. (Santiago: LOM ediciones, 2015), 254.

Bellei, Cristian; Cabalin, Cristian. Chilean Student Movements: Sustained Struggle to Transform a Market-Oriented Educational System. Current Issues in Comparative Education, v. 15, no. 2 (2013): 108-123.

Bellei, Cristian.; Valenzuela, Juan Pablo. “Están las condiciones para que la docencia sea una profesión de alto estatus en Chile?" Fin de ciclo. Cambios en la gobernanza del sistema educativo. (Santiago: UNESCO-PUC, 2010), 1-26.

Bravo, Andrés. "Historia y gestión del asentamiento de la Universidad Arturo Prat en la ciudad de Victoria" Documento de trabajo, no publicado, 2011.

Bravo, José. Educación pública, autonomía universitaria y cambio político: notas para el análisis del movimiento universitario en chile, 2011. Revista Historia de la Educación Latinoamericana, v.1, no.21 (2013): 263-282. https://doi.org/10.19053/01227238.2473

Cabalin, Cristian. "Neoliberal Education and Student Movements in Chile: Inequalities and Malaise". Policy Futures in Education, v. 10, no. 2 (2012): 219-228.

Comisión. "Informe comisión sobre formación inicial docente". (Santiago: CPEIP, 2005), 91. 
Contreras Gutiérrez, Alejandra. La enseñanza jesuita en Chile colonial: sus colegios, universidades y una aproximación a sus métodos y contenidos. Revista Historia de la Educación Latinoamericana, vol.16 no. 22 (2014): 35-50. https://doi.org/10.19053/01227238.2687.

Darling-Hammond, Linda y Lieberman, Ann. “Teacher education around the world: Changing policies and practices". (Nueva York: Routledge, 2013), 202.

De Castro, Sergio. El Ladrillo: bases de la política económica del gobierno militar chileno. (Santiago: Centro de Estudios Públicos,1992), 193.

Délano, Manuel y Traslaviña, Hugo. La herencia de los Chicago Boys. (Santiago: Ornitorrinco, 1989), 209.

Diniz-Pereira, Julio. "As licenciaturas e as novas políticas educacionais para a formação docente". Educação \& sociedade, v. 20, no. 68 (1990): 109-125.

Ganga, Francisco. A.; Vera, José. "Reflexiones en torno al nuevo escenario de la gestión universitaria". Cuadernos de la Facultad de Humanidades y Ciencias Sociales. Universidad Nacional de Jujuy, n. 30 (2006): 41-61.

Kornbluh, Peter. "The Declassified Pinochet File: Delivering the Verdict of History". Radical history review, v. 2016, n. 124 (2016): 203-216.

Kremerman, Marco. “El desalojo de la educación pública”. Serie "Ideas para el buen vivir". (Santiago:Fundación SOL. N 11, 2011), 13.

López, Ana. “De la dictadura militar a la democracia pactada de la Concertación y la derecha: El modelo de la Universidad-Empresa en Chile (1973-2008)". Lucha de clases, v. 1(2011).

Mineduc. Estadísticas de la educación 2016. (Santiago: Mineduc, 2017), 177.

Monckeberg, María Olivia. Privatización de las Universidades. (Santiago: Copa Rota, 2005),603.

Monckeberg, María Olivia. Con fines de lucro. (Santiago: Penguin Random House, 2013), 642.

Nef, Jorge. "The Chilean model: fact and fiction". Latin American perspectives, v. 30, n. 5 (2003): 16-40.

Nef, Jorge. "El concepto de Estado subsidiario y la educación como bien de mercado: Un bosquejo de análisis político". Enfoques educacionales, v. 2, (2007): 1-10.

OCDE. Establecimiento de un marco para la evaluación e incentivos docentes. Consideraciones para México. (Paris: OECD, 2011): 134.

Oliva, María Angélica. "Política educativa y profundización de la desigualdad en Chile". Estudios Pedagógicos (2008): 207-226.

Pino-Yancovic, Mauricio. “Parents' defense of their children's right to education: Resistance experiences against public school closings in Chile". Education, Citizenship and Social Justice, v. 10, no. 3, (2015): 254-265.

Reyes León, Daniel y Santos, Marcelo. “Los profesores taxis-El hedor de lo heredado: La desprofesionalización académica y su parque humano". Le Monde Diplomatique. Edición Chilena, 2011. revisado desde: https://www.lemondediplomatique.cl/LOS-PROFESORES-TAXIS-El-hedor-de.html

Rifo Melo, Mauricio "Historia de la transformación en la educación superior chilena (19731990)". Revista Historia de La Educación Latinoamericana, v. 19, no. 28, (2017), 13-36. doi. org/10.19053/01227238.4925

Ruffinelli, Andrea. Actualización de la sistematización de la oferta de Programas. Especiales de Pedagogía en Educación Básica de las Instituciones de Educación Superior Chilenas. Resumen Ejecutivo. (Santiago: CIDE, Universidad Alberto Hurtado, 2007): 1-243.

Ruffinelli, Andrea y Sepúlveda, Luis. "Sistematización de la oferta de programas especiales de pedagogía en educación básica de las instituciones de educación superior chilenas". Universidad Santiago: CIDE, Universidad Alberto Hurtado (2005): 1-29.

Simbürger, Elisabeth y Neary, Mike. "Taxi Professors: Academic Labour in Chile, a Critical-Practical Response to the Politics of Worker Identity". Workplace: A Journal for Academic Labor, v. 0, no. 28 (2016): 48-73.

Sisto, Vicente. “Entre la Privatización y La Reconstrucción de lo Público en Chile: Movimientos 
Estudiantiles y el Debate acerca del Devenir de la Universidad". Horizontes Sociológicos, v. 0, no. 1 (2015): 57-63.

Soto, Maximiliano. "Políticas Educacionales en Chile durante el Siglo XX". Revista Mad, v. 0, no. 10 (2004): 30-42.

Valdés, Juan Gabriel. La escuela de Chicago: operación Chile. (Buenos Aires: Grupo Editorial Zeta, 1989), 321.

Valdés, Juan Gabriel. Pinochet's Economists: The Chicago School of Economics in Chile. (New York: Cambridge University Press, 1995), 352.

Zeichner, Ken. "The Adequacies and Inadequacies of Three Current Strategies to Recruit, Prepare, and Retain the Best Teachers for All Students". Teachers College record, v. 105, 3 (2003): 490519.

Zeichner, Ken; Peña-Sandoval, César. "Venture philanthropy and teacher education policy in the US: The role of the New Schools Venture Fund". Teachers College record, v. 117, no. 6 (2015): $1-24$.

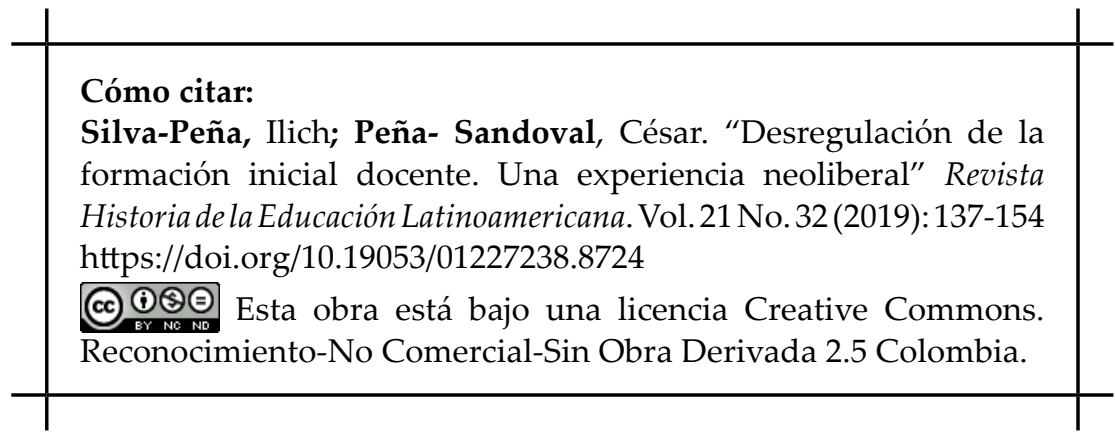

